\title{
TRANSPORTE DE MONÓXIDO DE CARBONO GERADO EM QUEIMADAS PARA REGIÕES ONDE NÃO SE QUEIMA
}

\author{
C.B. Aires \& V.W.J.H. Kirchhoff
}

Received May 11, 2000 / Accepted April 15, 2001

\begin{abstract}
Descreve-se uma campanha de medidas de monóxido de carbono (CO), a bordo de avião, para caracterizar a distribuição das concentrações numa região em que se observou um processo especial de transporte das massas de ar, que pode levar o produto das queimadas para regiões de pouca queima. O processo de queima injeta diretamente o $\mathrm{CO}$ na troposfera, próximo à superfície. Para quantificar a produção local de poluente, foi feito um levantamento quantitativo sobre os focos de queimada na superfície com base em dados de satélite. Um programa de cálculos de trajetória foi usado para informar o padrão de circulação de massas de ar na área de estudo. As trajetórias em destaque definem um "corredor de circulação de massas de ar", onde o transporte dos gases de queimada de regiões fonte para não fonte é privilegiado. Com estas informações e os dados adicionais de medidas de concentração de $\mathrm{CO}$, obteve-se as proporções de $\mathrm{CO}$, transportadas para diversas regiões na área da circulação estudada. $\mathrm{O}$ caso mais intenso mostra que na região ao norte de Mato Grosso do Sul e sul do estado de Goiás são injetados até 200 ppbv de CO (da ordem de $70 \%$ do valor observado), na época crítica. Já ao norte do estado do Paraná, $45 \%$ do valor observado de CO vem por transporte das regiões de maior índice de queima.
\end{abstract}

Palavras-chave: Monóxido de carbono; Queimadas; Gases de queimada; Focos de queimada.

TRANSPORT OF CARBON MONOXIDE FROM BIOMASS BURNING SOURCE TO NON-SOURCE REGIONS - An aircraft experiment is described that measured atmospheric carbon monoxide (CO) to characterize the distribution and transport of this fire product in a special air mass circulation region of central Brazil. Biomass burning directly injects CO into the lower troposphere, near the surface, and mixing and transport processes then distribute it to the whole troposphere. Satellite images of fire pixels were used to characterize the distribution of fires in the study area. Air mass trajectories were calculated in order to exactly determine the origin of the polluted air masses. Calculations are then made of the proportion of $C O$ concentrations that are observed at a certain point and which are the result of the transportation of this pollutant from source regions. In the northern part of the state of Mato Grosso, as well as the southern part of the state of Goias, for example, up to $200 \mathrm{ppbv}$ of CO (about $70 \%$ of the observed concentration) are the result of imports from stronger source regions. In the north of the state of Paraná, the imported amount of CO is about $45 \%$ of the observed value.

Key words: Carbon monoxide; Biomass burning; Pollutants; Fire pixels. 


\section{INTRODUÇÃO}

Em função da não uniformidade na distribuição espacial das queimadas, o Brasil apresenta grandes contrastes entre regiões fonte e não fonte de queimada. Esses contrastes são decorrentes de fatores climáticos (período de grande estiagem) e regionais (predominância de atividades agropecuárias). No entanto, nestas regiões de pouca queima, medem-se, às vezes, concentrações elevadas de gases traço gerados por queimadas. Em outras palavras, as regiões de muita queima, ou regiões fonte, exportam gases gerados em queimadas para regiões não fonte.

Por meio de cálculos de trajetória de massas de ar é possível determinar o caminho do transporte de massas de ar em altitudes definidas. Resultados obtidos através dessa técnica, evidenciam a existência de um "corredor de circulação de massas de ar" entre regiões fonte e não fonte de queimada, no continente brasileiro em baixas altitudes (cerca de 0 a $4 \mathrm{~km}$ ). Essas trajetórias foram determinadas de acordo com a metodologia utilizada pelo Centro de Previsão de Tempo e Estudos Climáticos do Instituto Nacional de Pesquisas Espaciais, CPTEC/INPE, de Cachoeira Paulista (SP), para cálculos de trajetória de massas de ar pelo método isentrópico, que será discutido posteriormente.

Assim, os principais objetivos deste trabalho são: 1- obter medidas aéreas originais de $\mathrm{CO}$ na região influenciada pelas queimadas sazonais do Brasil Central;

2- redefinir a região de circulação especial de massas de ar, usando cálculos de trajetória;

3- usando dados de focos de queimada do AVHRR/ NOAA, definir a produção local de CO;

4- determinar, finalmente, quanto da concentração observada é produzida localmente e quanto entra por transporte.

\section{Medidas anteriores de CO atmosférico no Brasil}

O primeiro trabalho realizado no Brasil de medidas de $\mathrm{CO}$ em superfície ou a bordo de avião foi realizado em uma expedição liderada pelo prêmio Nobel de química Paul Crutzen (Crutzen et al., 1985; Delany et al., 1985). Neste trabalho de campo um avião americano sobrevoou o território do Brasil
Central e mostrou os primeiros resultados de aumentos de concentração de $\mathrm{CO}$ por causa das queimadas no cerrado. Este trabalho pioneiro foi seguido por um trabalho observacional local (Kirchhoff \& Marinho, 1989; Kirchhoff et al., 1989; Kirchhoff \& Rasmussen, 1990; além de outros). Trabalhos posteriores confirmaram a sazonalidade da observação e mais do que isso, mostraram que durante uma certa época, de 1989 a 1995, estavam aumentando com o tempo (Kirchhoff, 1996). Um trabalho interessante de levantamento de concentrações a nível global foi feito pelo projeto MAPS, da NASA (Reichle et al., 1986) mas neste caso trata-se de uma integral de CO na troposfera média e alta.

\section{CARACTERIZAÇÃO DA ÁREA DE ESTUDO}

Por meio da técnica de cálculos de trajetória de massas de ar (que discutiremos com maiores detalhes mais adiante) é possível determinar qual a origem das massas de ar que chegam num determinado ponto de observação.

Utilizamos essa técnica para determinar qual o caminho que as massas de ar, provenientes do Oceano Atlântico, seguem no continente brasileiro, na baixa atmosfera. Assim, com base no estudo realizado, em que foram feitos vários cálculos de trajetória para a baixa atmosfera, constatamos que as parcelas de ar provenientes do Oceano Atlântico (relativamente isentas de contaminação) ao entrarem no continente brasileiro pela região nordeste prosseguem seus deslocamentos por regiões fonte de queimada no Brasil Central (região de Tocantins, Mato Grosso e Mato Grosso do Sul) e em virtude, em parte, da barreira topográfica natural, representada pela Cordilheira dos Andes, e em parte pela força do Anticiclone atmosférico, deixam o continente pela região sul-sudeste, que constitui uma área não fonte de queimada.

A Fig. 1 ilustra esses resultados. Nessa figura destacamos algumas cidades, como: Salvador - BA $\left(12^{0} \mathrm{~S}, 38^{0} \mathrm{~W}\right)$; Palmas - TO $\left(10^{0} \mathrm{~S}, 48^{\circ} \mathrm{W}\right)$, Cuiabá - MT $\left(16^{0} \mathrm{~S}, 54^{0} \mathrm{~W}\right)$; Campo Grande - MS $\left(20^{\circ} \mathrm{S}\right.$, $\left.54^{\circ} \mathrm{W}\right)$ e Curitiba - PR $\left(25^{\circ} \mathrm{S}, 49^{\circ} \mathrm{W}\right)$ a fim de melhor caracterizar esse corredor de circulação de massas de ar (Oceano Atlântico - Região Central do 


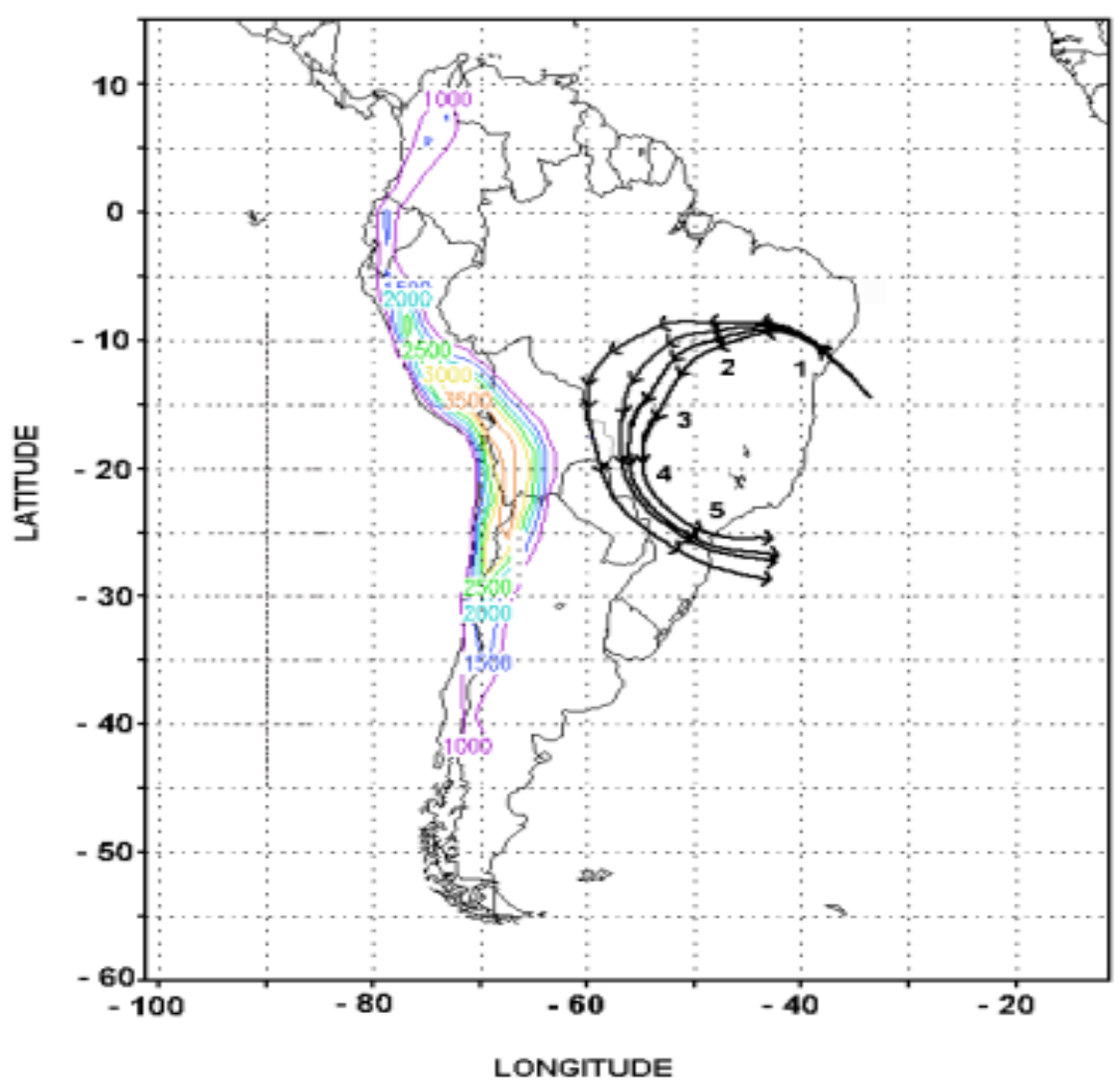

Figura 1 - Trajetórias para frente para o período de 9 dias (24/07 a 01/08/98) em superfícies isentrópicas com temperaturas potenciais de 307,5; 308,0; 308,5 e 309,0 K centralizando a região do corredor de massas de ar. Regiões importantes são: 1 . Salvador (12 $\left.{ }^{0} \mathrm{~S}, 38^{0} \mathrm{~W}\right) ; 2$. Palmas $\left(10^{\circ} \mathrm{S}, 48^{\circ} \mathrm{W}\right)$, 3. Cuiabá $\left(16^{0} \mathrm{~S}, 54^{\circ} \mathrm{W}\right)$; 4. Campo Grande $\left(20^{\circ} \mathrm{S}, 54^{\circ} \mathrm{W}\right)$; e 5. Curitiba $\left(25^{\circ} \mathrm{S}, 49^{\circ} \mathrm{W}\right)$.

Figure 1 - Map of South America showing an example of calculated forward trajectories for the period 24 July to 01 August, on isentropic surfaces with potential temperatures of 307,5; 308,0; 308,5 and 309,0 K defining the special circulation pattern of the present study. Important cities that are affected are, for example: 1 . Salvador - BA $\left(12^{\circ} \mathrm{S}, 38^{\circ} \mathrm{W}\right) ; 2$. Palmas - TO $\left(10^{\circ} \mathrm{S}, 48^{\circ} \mathrm{W}\right), 3 . \mathrm{Cuiaba}-\mathrm{MT}\left(16^{0} \mathrm{~S}\right.$, $\left.54^{0} \mathrm{~W}\right)$; 4. Campo Grande - MS (20 $\left.\mathrm{S}, 54^{\circ} \mathrm{W}\right)$; and 5. Curitiba - PR $\left(25^{\circ} \mathrm{S}, 49^{\circ} \mathrm{W}\right)$.

Brasil-Região Sul-Oceano Atlântico) no continente brasileiro, que define linhas de fluxo concêntricas que ao passarem por regiões fonte de queimada são enriquecidas por contaminantes produzidos in situ, sendo então transportadas, em geral, num curto intervalo de tempo (período da ordem de 10 dias), para outras regiões não fonte. Nesse trajeto as parcelas de ar mantiveram em altitudes entre 2,0 a $3,5 \mathrm{~km}$.

\section{Meteorologia da Área de Estudo}

Do ponto de vista meteorológico, o corredor de circulação de massas de ar identificado pelos cálculos de trajetória de massas de ar, representa um padrão típico de circulação no continente brasileiro (troposfera) para o período de inverno. Segundo
Nobre et al. (1998) as principais características do sistema de circulação de larga escala na baixa troposfera que atuam na América do Sul durante o inverno são: a Zona de Convergência Intertropical (ITCZ) sobre os oceanos Atlântico e Pacífico, associada às atividade convectiva no noroeste da América do Sul (Colômbia, Venezuela) e América Central; os sistemas transientes frontais (FSs) associados às frentes frias na América do Sul temperada e subtropical; e o Sistema de Alta Pressão Subtropical do Oceano Atlântico (SASH) ou também chamado Anticiclone do Atlântico Sul.

Durante o inverno ocorre o deslocamento para oeste (em direção ao continente) do SASH e o movimento para o norte da ITCZ, que produzem uma subsidência de larga escala típica sobre o Brasil Central e a Amazônia, deslocando umidade e nuvens 
para áreas remotas ao norte e noroeste da Amazônia. Esses fenômenos são responsáveis pela escassez de chuvas sobre o continente e pela definição da estação seca no Brasil Central (Longo et al., 1999; Vianello \& Alves 1991).

O deslocamento do SASH em direção ao continente favorece a entrada de massas de ar provenientes do oceano, pela porção nordeste do Brasil. As massas de ar provenientes do Oceano Atlântico relativamente isentas de contaminação ao entrar no continente brasileiro, passam por regiões fonte de queimada no Brasil Central (bioma cerrado), que por meio de um escoamento anticiclônico no continente deixam o continente pela região sulsudeste. A Fig. 2 (Satyamurty et al., 1998) mostra uma configuração típica média dos ventos para o mês de julho em $850 \mathrm{hPa}$ (cerca de 2,5 km de altitude), que evidencia o padrão de circulação descrito no exemplo da Fig. 1.

\section{INSTRUMENTOS E MÉTODOS}

\section{Medidas de CO pela técnica de cromatografia gasosa}

A técnica de medida de concentrações de monóxido de carbono (CO), usada neste trabalho, é

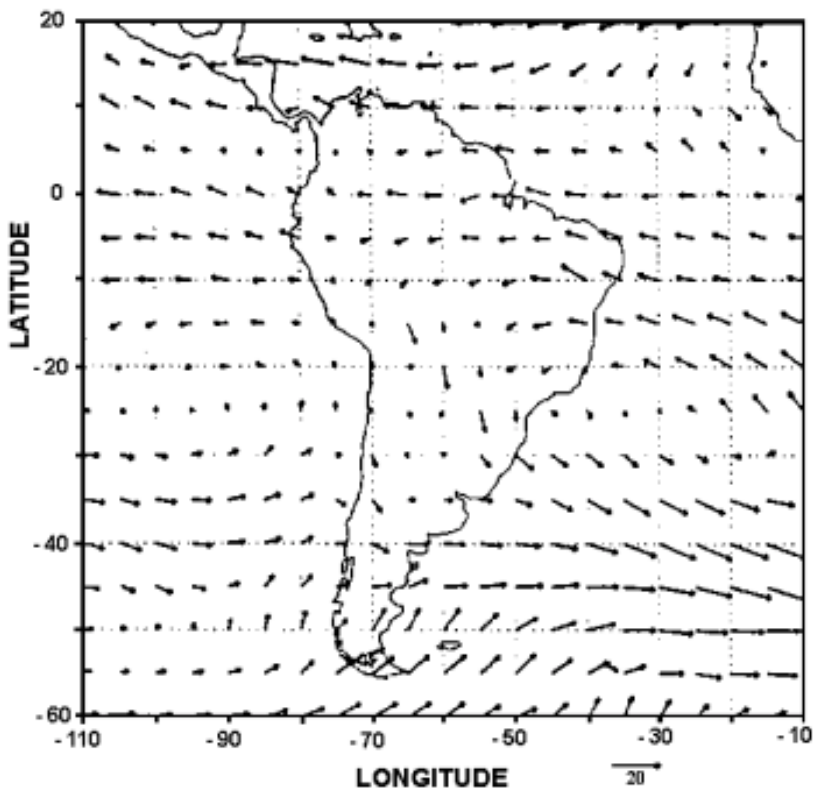

Figura 2 - Distribuição típica do vetor vento médio $\left(\mathrm{m} \mathrm{s}^{-1}\right)$ para julho, em 850 hPa, segundo Satyamurti et al. (1998).

Figure 2 - Typical mean wind vector $\left(\mathrm{m} \mathrm{s}^{-1}\right)$ distribution at 850 hPa for July, according to Satyamurti et al. (1998). descrita na literatura por Seiler \& Junge (1970) e Seiler (1974), e se baseia no método de separação cromatográfica seguida por detecção de vapor de mercúrio. Sendo mundialmente utilizada,essa técnica apresenta boa resolução e precisão de $0,2 \%$, bem como boa linearidade de resposta (Mariano, 1992).

As amostras de ar são coletadas por uma pequena bomba de sucção, que pressuriza garrafas de aço inoxidável, especialmente construídas para esse propósito. Essas garrafas tem capacidade de 800 $\mathrm{ml}$ e são eletropolidas internamente, a fim de evitar a fixação de moléculas de gases nas paredes. A análise de uma dessas garrafas pelo método de cromatografia é feita em cerca de 5 minutos (Kirchhoff \& Marinho, 1988; 1990). A amostra a ser analisada é injetada no módulo cromatográfico, na coluna de amostragem, que tem $2 \mathrm{ml}$ de volume. Essa coluna de amostragem é inserida em série com a coluna de separação, através de uma válvula de seis vias, e a amostra é então levada à coluna de separação pelo gás de arraste (ar sintético). O gás de arraste é seco e filtrado para eliminar possíveis interferências de hidrocarbonetos e dióxido de enxofre $\left(\mathrm{SO}_{2}\right)$, que também podem reagir com o óxido de mercúrio. A concentração de $\mathrm{CO}$ na amostra é determinada por comparação com os gases de calibração (Kirchhoff \& Rasmussen, 1990).

A coluna de separação é a coluna cromatográfica propriamente dita, onde os diversos componentes da amostra são separados por adsorção temporária, nas partículas do material da coluna, mantida à temperatura de $80^{\circ} \mathrm{C}$ (processo de separação cromatográfica). Os gases são então dirigidos a um segundo módulo, que tem a função de detectar o CO. Os gases passam primeiro pela câmara reatora onde uma camada de $\mathrm{HgO}$ entra em contato com os gases da amostra a uma temperatura de $160{ }^{\circ} \mathrm{C}$. É nesse reator que o

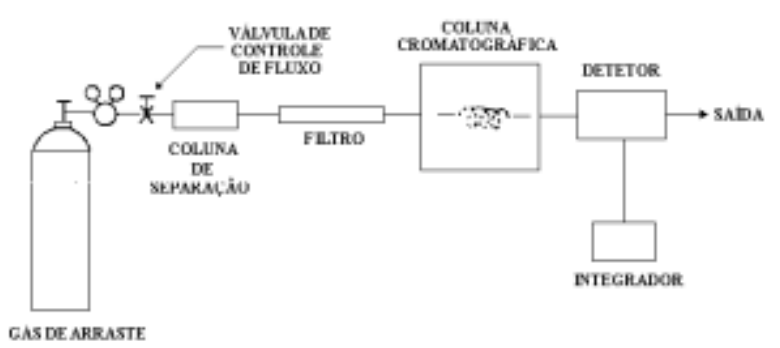

Figura 3 - Diagrama esquemático do cromatógrafo que mede a concentração do $\mathrm{CO}$.

Figure 3 - Schematic representation of chromatography used to measure CO concentrations. 
$\mathrm{CO}$ é transformado em $\mathrm{CO}_{2}$, liberando o $\mathrm{Hg}$ no estado de vapor.

O vapor de mercúrio é medido através de um sistema óptico, onde ocorre a absorção da radiação UV de uma lâmpada de $\mathrm{Hg}$, sendo que a radiação atenuada pelo vapor de $\mathrm{Hg}$ é proporcional à quantidade de $\mathrm{CO}$ presente na amostra. $\mathrm{O}$ resultado é enviado a um monitor (ver esquema na Fig.3) que é em geral um registrador de pena, um integrador, ou um computador dedicado (Kirchhoff \& Marinho, 1988). A unidade de medida é partes por bilhão por volume (ppbv).

Além das medidas de $\mathrm{CO}$, obtidas na plataforma do avião, fazem-se rotineiramente também medidas de $\mathrm{O}_{3}, \mathrm{CH}_{4}$, e $\mathrm{N}_{2} \mathrm{O}$, mas a ênfase neste trabalho é o CO. Para as medidas in situ de ozônio de superfície usam-se fotômetros de absorção de UV. Estes instrumentos tem sido usados mundialmente para medir ozônio troposférico (Kirchhoff et al.1989; 1990). $\mathrm{O} \mathrm{CO}_{2}$ é medido continuamente por absorção infra-vermelha do tipo não dispersivo. Estes instrumentos foram usados na plataforma de vôo do avião Bandeirante de pesquisa do INPE para obter perfis dos gases traço entre 1 e $6 \mathrm{~km}$ de altitude (Kirchhoff \& Alvalá, 1996; ver descrição adicional adiante).

O avião do INPE é um bi-motor tipo Bandeirante, modelo EMB 110, utilizado na realização de medidas de gases traço na atmosfera. Por não ser pressurizado seu uso limita-se a altitudes inferiores a $5 \mathrm{~km}$. Para casos especiais pode-se usar máscaras de oxigênio, mas isto impõe um esforço adicional aos operadores, e por isso é evitado (Kirchhoff \& Alvalá, 1996). Os equipamentos para medidas de gases traço são montados num armário de aço tipo rack que posteriormente é fixado no avião. As amostras de ar são conduzidas aos medidores, por entradas de ar conectadas na parte externa da aeronave que com auxílio de uma bomba à vácuo realiza a sucção do ar para dentro da instrumentação.

\section{Cálculos de trajetória de Massas de Ar}

No estudo da presença de contaminantes atmosféricos é muito importante o conhecimento da procedência (origem) das massas de ar e seus deslocamentos, pois a presença de um poluente atmosférico numa região, pode ser devido à produção in situ e/ou a processos de transportes.

Do ponto de vista da dinâmica, o conhecimento dos ventos no local de estudo não é suficiente para a determinação da origem das massas de ar, porque o vetor velocidade em parte é devido a movimentos de rotação, e não de translação das massas de ar. É preciso conhecer a trajetória seguida pelas mesmas e somente através do conhecimento dinâmico da atmosfera é que podemos mapear esses deslocamentos. Assim, uma ferramenta importante para o estudo de transporte dos contaminantes atmosféricos, em diferentes níveis de pressão, tem sido o cálculos de trajetória de massas de ar em função do tempo (Doty \& Perkey, 1993).

Esses cálculos baseiam-se em parâmetros de entrada tais como: campos de ventos (horizontal e vertical), altitude geopotencial, temperatura potencial, níveis de pressão, valores estes que correspondem a dados provenientes de estações de superfície, navios, bóias, balões climáticos e satélites, obtidos através de grandes centros meteorológicos, como por exemplo, o National Center for Environmental Prediction - NCEP; na utilização de programas computacionais para a resolução das equações de movimento das parcelas de ar e na aplicação de técnicas de interpolação para o cálculo dos valores desconhecidos em pontos irregulares (Austin \& Tuck, 1985; Merril et al., 1986).

Existem diferentes modelos de trajetórias: isentrópicos, isobáricos ou cinéticos. No modelo isentrópico, as trajetórias são calculadas com base no deslocamento das parcelas de ar sobre superfícies isentrópicas (superfícies de mesma temperatura potencial) num movimento adiabático. No isobárico o deslocamento das parcelas é avaliado sobre superfícies com pressão constante. O modelo cinético requer informações sobre o campo de velocidades verticais (Kahl et al., 1989).

Uma incerteza associada a cálculos de trajetória deve-se à técnica utilizada. Trajetórias isentrópicas tem sido amplamente utilizadas (Pickering et al., 1994, 1996) e tem a vantagem de não requerer a velocidade vertical do vento. $O$ formalismo cinemático utiliza as componentes do campo de vento para o cálculo de trajetória. Fuelberg et al. (1996) durante o experimento Transport and Atmospheric Chemistry 
Near the Equator-Atlantic (TRACE A), calcularam trajetórias cinemáticas e isentrópicas integrando-as por um período de cinco dias (13-18 de outubro de 1992 utilizando análises do ECMWF). Seus resultados mostraram que as trajetórias calculadas pelo método cinemático, usualmente possuem um deslocamento vertical maior que as correspondentes isentrópicas. Segundo Fuelberg et al. (1996) ambos os formalismos isentrópico e cinemático podem fornecer trajetórias realistas, na troposfera, com integração de até 5 dias.

Com relação ao tempo, as trajetórias podem ser analisadas para frente ("forward") ou para trás ("backward"). A primeira considera uma parcela inicial no tempo-espaço e acompanha esse deslocamento no sentido positivo do tempo até a sua posição final, ao contrário da segunda que acompanha o deslocamento no sentido negativo do tempo até a posição de origem (Doty \& Perkey, 1993). Assim, com o cálculos de trajetória para frente podemos inferir para onde uma parcela de ar está se deslocando a partir de um ponto inicial de observação, ao contrário do resultado obtido para o cálculos de trajetória para trás que permite inferir qual a procedência das massas de ar que estão chegando num determinado ponto de observação. Nos exemplos a seguir usa-se cálculos de trajetória isentrópica, segundo o método desenvolvido pelo especialista Dr. Igor Trosnikov do CPTEC/INPE. Os parâmetros de entrada: campos de ventos (horizontal e vertical), altitude geopotencial, temperatura potencial, e níveis de pressão, foram obtidos do NCEP, cujos arquivos de dados possuem o seguinte formato (NCEP, 1998; NCEP, HYPERLINK, http://wesley.wwb.noaa.gov// ncep_data/index.html; Dados meteorológicos, 1998). a) 17 níveis de pressão $=(1000 ; 925 ; 850 ; 700 ; 600$; $500 ; 400 ; 300 ; 250 ; 200 ; 150 ; 100 ; 70 ; 50 ; 30 ; 20 \mathrm{e}$ 10 mbar );

b) resolução espacial: 2,50 x 2,50 (latitude $\mathrm{x}$ longitude);

c) formato binário.

O método de interpolação polinomial usado foi o de Williamson \& Rasch (1989); Conte \& Boor (1981) e o programa utilizado para a visualização das

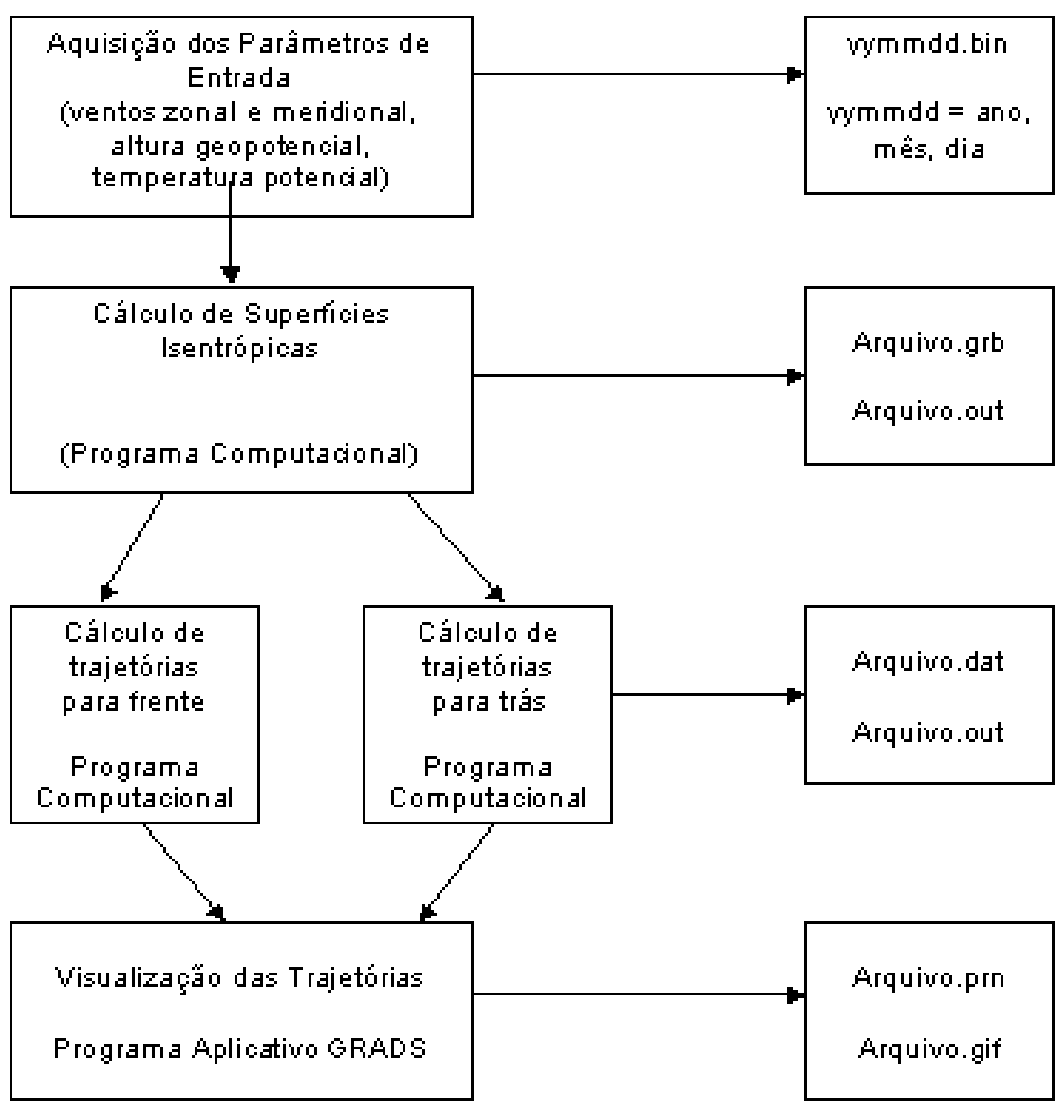

Figura 4 - Esquema da rotina para os cálculos de trajetória para o modelo isentrópico adotado neste trabalho.

Figure 4 - Schematic representation of the trajectory calculations routine, using an isentropic model. 
trajetórias foi o Grid Analysis and Display System, GRADS, que consiste num programa aplicativo proveniente dos Estados Unidos, University of Maryland, Center for Ocean Land-Atmosphere Interactions, Department of Meteorology muito utilizado na área de meteorologia e ciência da Terra (Doty, 1995). A Fig.4 mostra a rotina utilizada para os cálculos de trajetória para a frente e para trás pelo método isentrópico adotado.

\section{Detecção de Queimadas pelo Sistema Imageador AVHRR/NOAA}

Considerando a extensão territorial do país e a relativa falta de condições de controle e fiscalização do fogo, a utilização do sensoriamento remoto orbital, para a detecção e monitoramento de queimadas no Brasil tem se mostrado uma técnica útil. Através de imagens termais (faixa de 3,55 - 3,95 $\mathrm{mm}$ ) diárias dos satélites meteorológicos da série NOAA (National Oceanic and Atmospheric Administration), tem sido possível, desde 1987, a detecção de queimadas

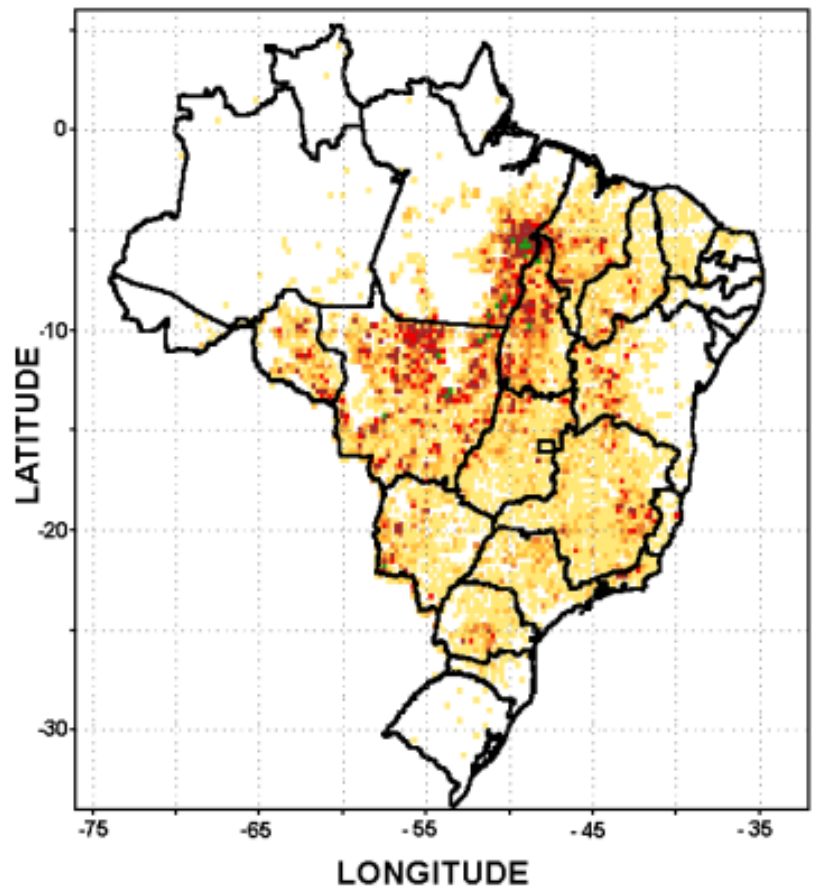

Figura 5 - Mapa do Brasil com a distribuição espacial dos focos de queimada segundo o AVHRR do NOAA 12, para o mês de setembro de 1999 .

Figure 5 - Map of Brazil showing the division of states, and the spatial distribution of fire pixels for the month of September, 1999; AVHRR infrared channel data from NOAA 12. (focos de calor) em tempo quase-real para o período de 01 de maio a 30 de novembro, época de estiagem no Brasil Central e Sul da Amazônia. Desde agosto de 1995, a obtenção dessas imagens tem sido feita pelos satélites NOAA 12 e 14 (Setzer et al. 1992). O INPE possui na cidade de Cachoeira Paulista (SP), uma estação receptora de imagens AVHRR (Very High Resolution Radiometer) sistema operado pela NOAA. O sistema de detecção de queimadas a partir da imagem AVHRR em uso operacional no INPE foi de grande importância para este trabalho.

A Fig.5 corresponde ao produto final da análise de focos de queimada, proveniente dos satélite NOAA 12, para o mês de setembro de 1999 e representa a distribuição espacial das queimadas no Brasil para o referido período (consultas para CPTEC, http://www.cptec.inpe.br; Mapa de focos de queimada, 1999). O original fornecido pelo INPE é colorido. Neste caso mostra-se uma versão mais simples, em preto e branco, porque o objetivo é apenas mostrar a distribuição relativa dos focos de queimada.

Com base na distribuição dos focos de queimada por mês para cada região brasileira para vários anos, assim como no exemplo acima, podemos visualizar uma distribuição quase que padrão dos focos por mês e o enorme contraste que existe no número de focos entre a região centro-oeste e a sul, por exemplo. Esta não uniformidade na distribuição dos focos de queimada por região caracteriza a existência de áreas fonte e não fonte de queimada no Brasil, como já comentado.

Assim para atingirmos o objetivo de determinar quanto da concentração observada é produzida localmente e quanto entra por transporte foram adotadas as seguintes estratégias: medidas aéreas de CO para determinar a concentração total local, cálculos de trajetória de massas de ar, para redefinir a região de circulação especial de massas de ar e análise dos dados de focos de queimada para a determinação da produção local (regional) de $\mathrm{CO}$; os resultados obtidos com o auxílio dessas ferramentas serviram de subsídio para a construção de um modelo para a determinação de que percentual da concentração total medida é devido ao transporte (termo de transporte). 


\section{RESULTADOS E DISCUSSÕES}

\section{Medidas de CO}

No período de 18 a 21 de setembro de 1999 realizou-se um vôo com o avião Bandeirante do INPE ao longo da área do corredor de circulação de massas de ar onde foram realizadas medidas de monóxido de carbono para altitudes entre 2,0 a 2,5 km (região de melhor definição desse corredor). Objetivamos com essa campanha ter uma idéia do comportamento e distribuição das concentrações de monóxido de
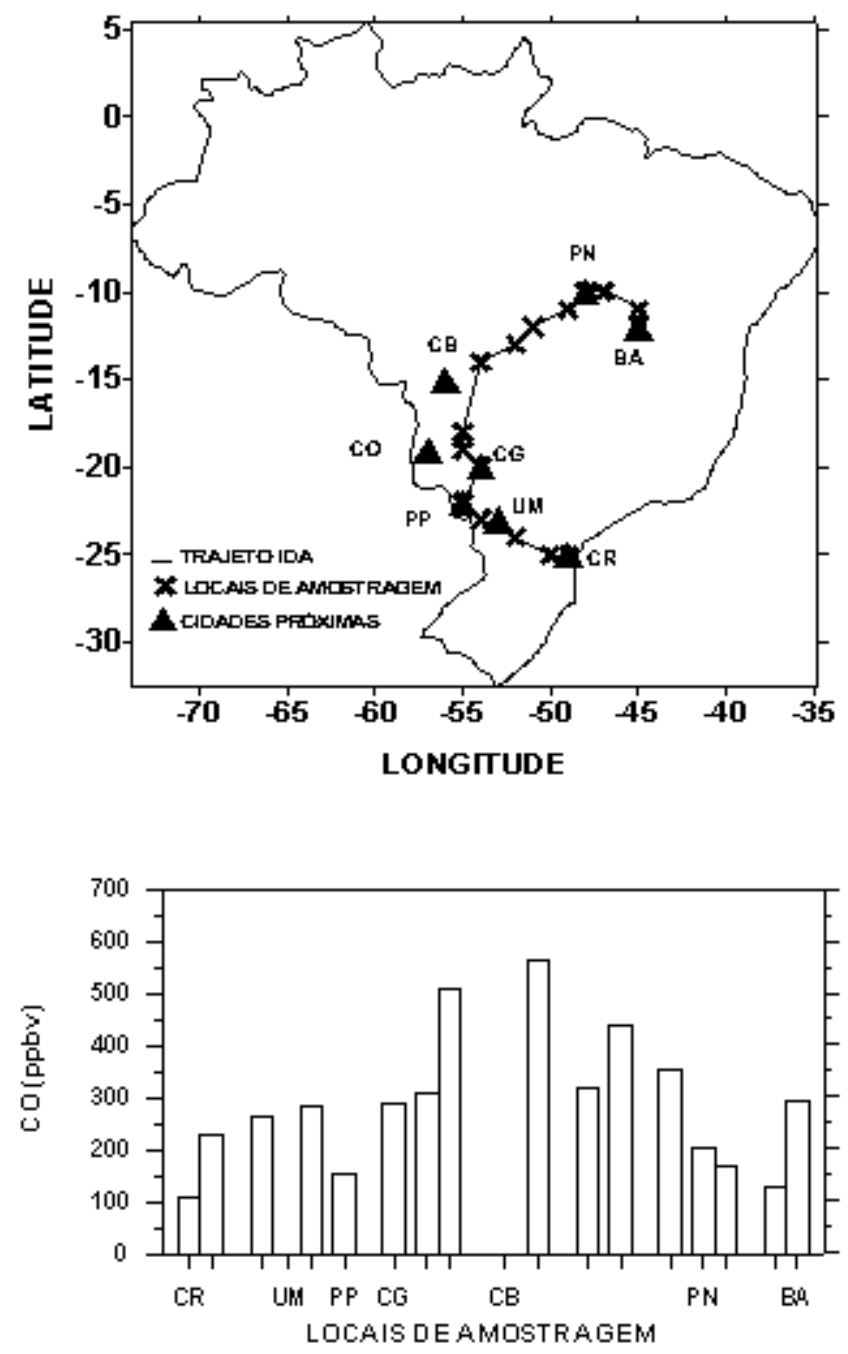

Figura 6 - Trajeto de ida da amostragem ao longo do corredor de circulação de massas de ar e as concentrações de CO medidas, no vôo com o avião Bandeirante do INPE, período de 18 a 21 de setembro de 1999.

Figure 6 - Flight track of the aircraft experiment and the observed CO concentrations, for the period of September 18 to 21, 1999. carbono ao longo das linhas de fluxo de massas de ar identificadas pelos cálculos de trajetória de massas de ar. As medidas foram feitas durante os vôos de ida (trajeto Curitiba - Barreiras) e de volta (trajeto Barreiras-Curitiba).

As amostras de $\mathrm{CO}$ foram coletadas em garrafas de aço inoxidável próprias para esse fim, para posterior análise, pelo método de cromatografia gasosa, no Laboratório de Ozônio do INPE, conforme metodologia descrita anteriormente. Ao todo foram coletadas 30 garrafas ao longo da área de estudo.
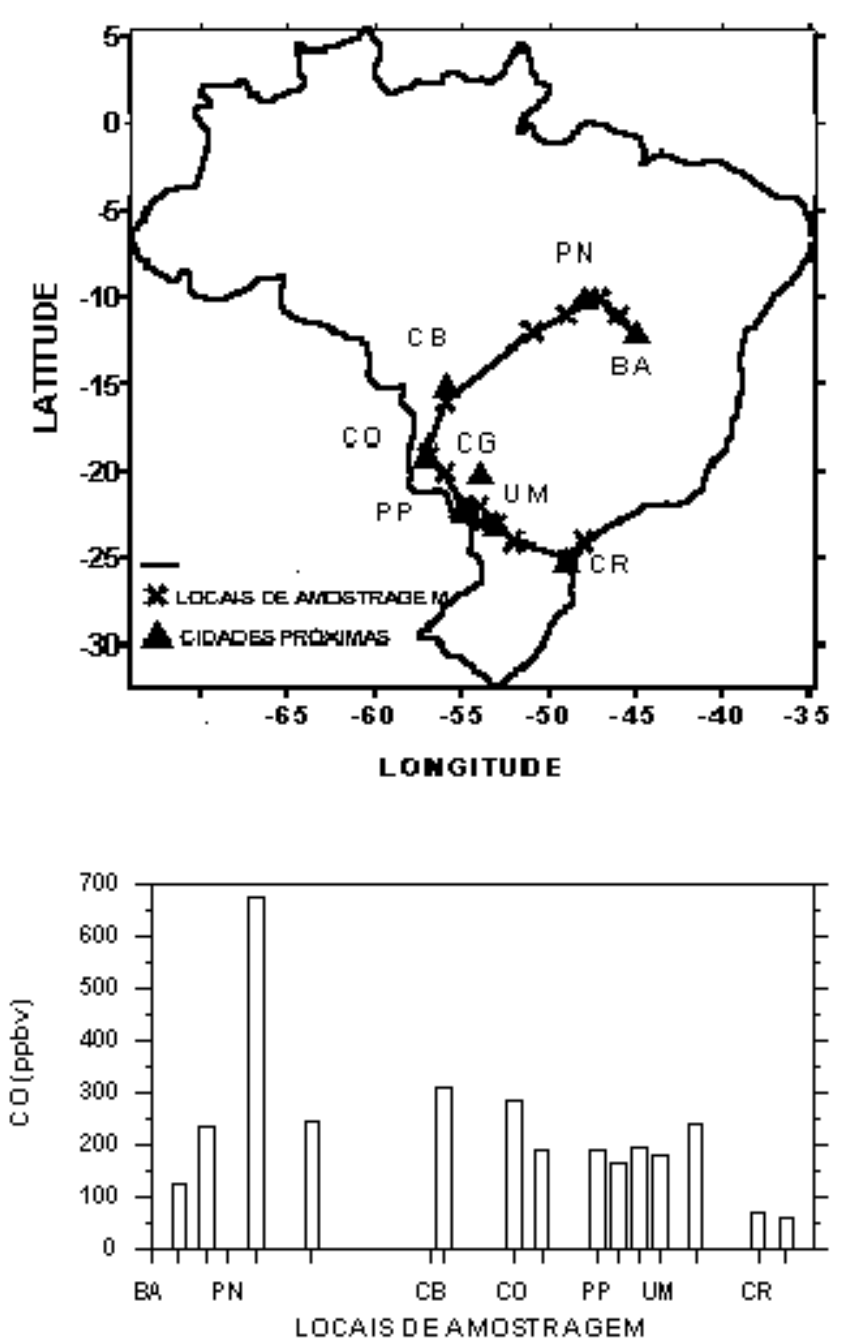

Figura 7 - Trajeto de volta da amostragem ao longo do corredor de circulação de massas de ar e as concentrações de CO medidas, no vôo com o avião Bandeirante do INPE, período de 18 a 21 de setembro de 1999.

Figure 7 - Flight track (return legs) of the aircraft experiment and the observed CO concentrations, for the period of September 18 to 21,1999 . 
As Figuras 6 e 7 mostram, respectivamente, as concentrações obtidas ao longo da área de estudo nos trajetos de ida (Curitiba-Barreiras) e de volta (Barreiras-Curitiba). As siglas utilizadas nas referidas figuras correspondem aos nomes das principais cidades ao longo do trajeto. Assim, temos:

$\mathrm{BA}=$ Barreiras $(\mathrm{BA}) ; \mathrm{CB}=$ Cuiabá $(\mathrm{MT}) ; \mathrm{CG}$ $=$ Campo Grande (MS); $\mathrm{CO}=$ Corumbá $(\mathrm{MT}) ; \mathrm{CR}$ $=$ Curitiba $(\mathrm{PR}) ; \mathrm{UM}=\mathrm{Umuarama}(\mathrm{PR}) ; \mathrm{PN}=$ Porto Nacional (TO) e PP = Ponta Porã (MS).

Com os resultados obtidos nessa campanha observou-se um gradiente de concentrações de $\mathrm{CO}$, com valores máximos em torno de regiões fonte de queimada (Porto Nacional/TO; Cuiabá/MT) diminuindo gradualmente no sentido de regiões não fonte (ver Figs. 6 e 7), evidenciando uma boa relação entre aumentos nas concentrações de $\mathrm{CO}$ e números elevados de focos de queimada (dados de satélite).

\section{CÁLCULOS DO TERMO DE TRANSPORTE}

Nesta seção apresentam-se os cálculos para a determinação do termo de transporte de contaminantes atmosféricos, trazidos de regiões fonte

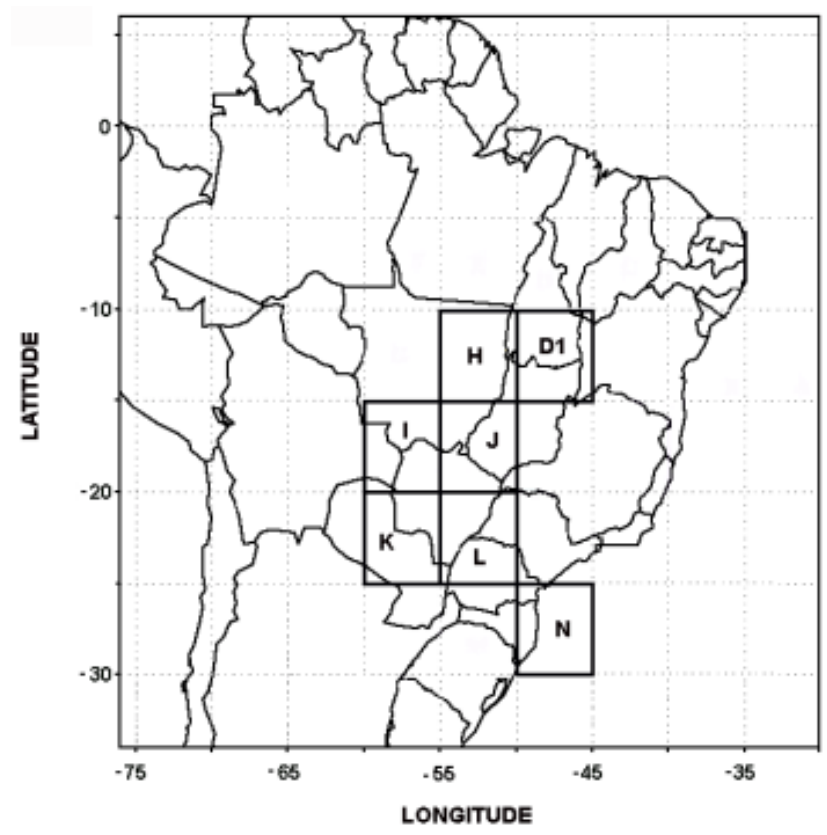

Figura 8 - Mapa do Brasil com a subdivisão da área do corredor de circulação de massas de ar em células de $5^{0}$ x $5^{0}$, designadas pelas letras D1, H, I, J, K, L e N.

Figure 8 - Map of Brazil showing the adopted division of the region of special air mass circulation in grid cells of $5^{0} X 5^{0}$ indicated by $D 1, H, I, J, K, L$ and $N$. para regiões não fonte, desenvolvidos a partir de um formalismo matemático baseado na equação da continuidade e de dados obtidos experimentalmente (medidas da concentração de $\mathrm{CO}$ e dados de satélite de focos de queimada). A fim de simplificarmos a análise da área de estudo gradeamos a região de interesse em subáreas de $5^{0} X 5^{0}$, que chamamos de células, e identificamos pelas letras D1, H, I, J, K, L, e N, mostradas na Fig.8.

Alguns fatores importantes que devem garantir a eficiência da metodologia descrita para a análise dos termos de transporte são: o fato do gás traço $\mathrm{CO}$ utilizado para esse estudo ser o resultado imediato da queima incompleta de biomassa e ter vida média longa (Novelli et al., 1998), o que nos possibilita desconsiderar em nossa análise o termo de perda da equação da continuidade; o padrão de circulação climática de inverno da área de estudo que define um corredor de circulação de massas de ar garantindo que haja um fluxo de massas de ar contínuo entre regiões fonte e não fonte de queimada, no período de queima de biomassa e o regime de larga subsidência que favorece a ocorrência de queimadas no Brasil Central e portanto a manutenção das fontes produtora.

Com base nos princípios de continuidade, determinamos a equação básica a ser aplicada em cada célula para a determinação do termo de transporte. Conforme pode ser demonstrado, a concentração total medida num ponto é devida a contribuições de três termos: residual, produção regional e a contribuição de transporte (isto é, a parte que vem de outras regiões). Assim, temos:

$$
\mathrm{Cm}=\mathrm{Cb}+\mathrm{Cr}+\mathrm{Ct}
$$

onde:

$\mathrm{Cm}=$ Concentração medida;

$\mathrm{Cb}=$ Concentração residual;

$\mathrm{Cr}=$ Concentração regional;

$\mathrm{Ct}=$ Concentração de transporte.

As concentrações medidas $\mathrm{Cm}$ correspondem às medições feitas neste projeto, ao longo da região do corredor de circulação de massas de ar. Para as medidas feitas a bordo do avião, usamos altitudes entre 2,0 a 2,5 km (altitudes em que, de acordo com os cálculos de trajetórias de massas de ar, o corredor de circulação de massas de ar encontra-se melhor 


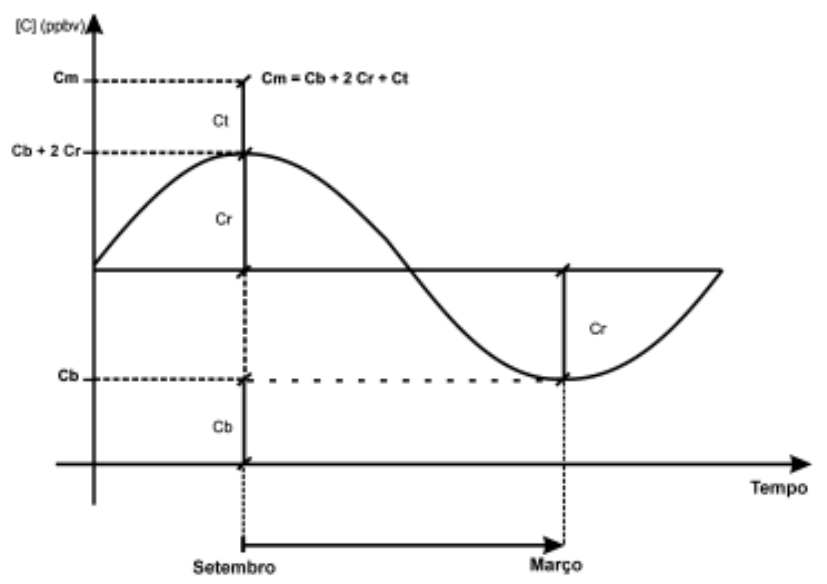

Fig. 9 - Diagrama esquemático para indicar a variação temporal da contribuição regional $\left(\mathrm{C}_{\mathrm{r}}\right)$ e definição dos termos $\mathrm{C}_{\mathrm{m}}, \mathrm{C}_{\mathrm{b}}$ e $\mathrm{C}_{\mathrm{t}}$.

Fig. 9 - Schematic representation of the temporal variation of the regional contribution $C_{r}$, and definition of the terms $C_{m}, C_{b}$ and $C_{t}$

definido). Nesta faixa de altitudes, experimentos anteriores mostram que as razões de mistura do $\mathrm{CO}$ variam pouco (Kirchhoffe Alvalá, 1996). O uso de avião possibilita a obtenção, praticamente simultânea, de concentrações de CO ao longo da área de estudo. Mesmo supondo que num dado local não haja produção nenhuma de contaminantes e que não estejam sendo transportados contaminantes de outras regiões, existe uma concentração mínima denominada de residual, como indicado na Fig.9. Em nosso estudo o termo residual corresponde a um valor médio de concentrações mínimas observadas durante a ausência de queima de biomassa (período de chuvas). De acordo com experimentos anteriores e dados da literatura, concentrações médias de $\mathrm{CO}$ entre $80 \mathrm{e}$ 100 ppbv são valores médios típicos da porção residual (Kirchhoff \& Alvalá, 1996; Sachse et al., 1988).

A Fig.9 mostra o esquema básico de nossa simulação da variação anual dos contaminantes de queimada. A curva mostra um valor medido genérico de concentração $\mathrm{Cm}$, representado para o mês de máximo, setembro. A curva senoidal representa a variação temporal da contribuição regional (mas o valor de fato é o observado pelo sensor a bordo do satélite); o valor de base representa $\mathrm{Cb}$, e o termo de transporte (incógnita procurada) está representado por Ct. Nota-se que no limite, quando a contribuição regional é nula, e o transporte também, o valor de $\mathrm{Cm}$ se confunde com o de $\mathrm{Cb}$. No presente caso, a região de interesse foi dividida em células, para as quais foi obtido o número de focos de queimada.

Define-se o termo de contribuição regional, por exemplo para o mês de setembro, como sendo:

$$
\mathrm{Cr}=2 \mathrm{NF}
$$

onde:

N é o número de focos de queimada por célula; e F é um fator de conversão, dimensional, de focos de queimada para concentração de $\mathrm{CO}$ (ppbv).

Com base nos valores medidos de $\mathrm{CO}$ e por meio de programa computacional desenvolvido, calculamos o termo de transporte $\mathrm{Ct}$, e ainda testamos a "convergência" do formalismo desenvolvido para o cálculo do termo de transporte, variando os parâmetros de entrada dentro de uma certa faixa e lógica. Objetivamos com isso testar a sensibilidade do programa em função da variação dos parâmetros de entrada. Assim, consideramos algumas suposições:

Três casos de condições de contorno para a célula D1, são apresentadas onde variamos por hipótese o valor do termo de transporte inicial, na primeira região (célula D1 da Fig. 8 = região de Tocantins), por onde passam as massas de ar. Assim, temos os seguintes desdobramentos: Hipótese de que $\mathrm{CtD} 1=0$ (supondo que as massas de ar vindas do Oceano Atlântico estão isentas de contaminação, portanto inicialmente não transportam contaminantes para a primeira célula);

Hipótese $\mathrm{CtD} 1=25$ ppbv (supondo que as massas de ar vindas do Oceano Atlântico transportam uma pequena concentração de contaminantes para a primeira célula); e Hipótese $\mathrm{C}, \mathrm{CtD} 1=50 \mathrm{ppbv}$ (supondo que as massas de ar vindas do Oceano Atlântico transportam uma concentração significativa de contaminantes para a primeira célula). Os gráficos comparativos entre os termos de transporte por célula para as diferentes hipóteses são apresentados na Fig. 10 , onde os três painéis representam as três condições de teste:

$$
\begin{aligned}
& \mathrm{Cb}=100 \text { ppbv; } \\
& \mathrm{Cb}=80 \text { ppbv; } \\
& \mathrm{Cb}=60 \text { ppbv. }
\end{aligned}
$$

As ordenadas na Fig. 10 mostram os valores de concentração oriundos do transporte de contaminantes (resultado das hipóteses assumidas) e 

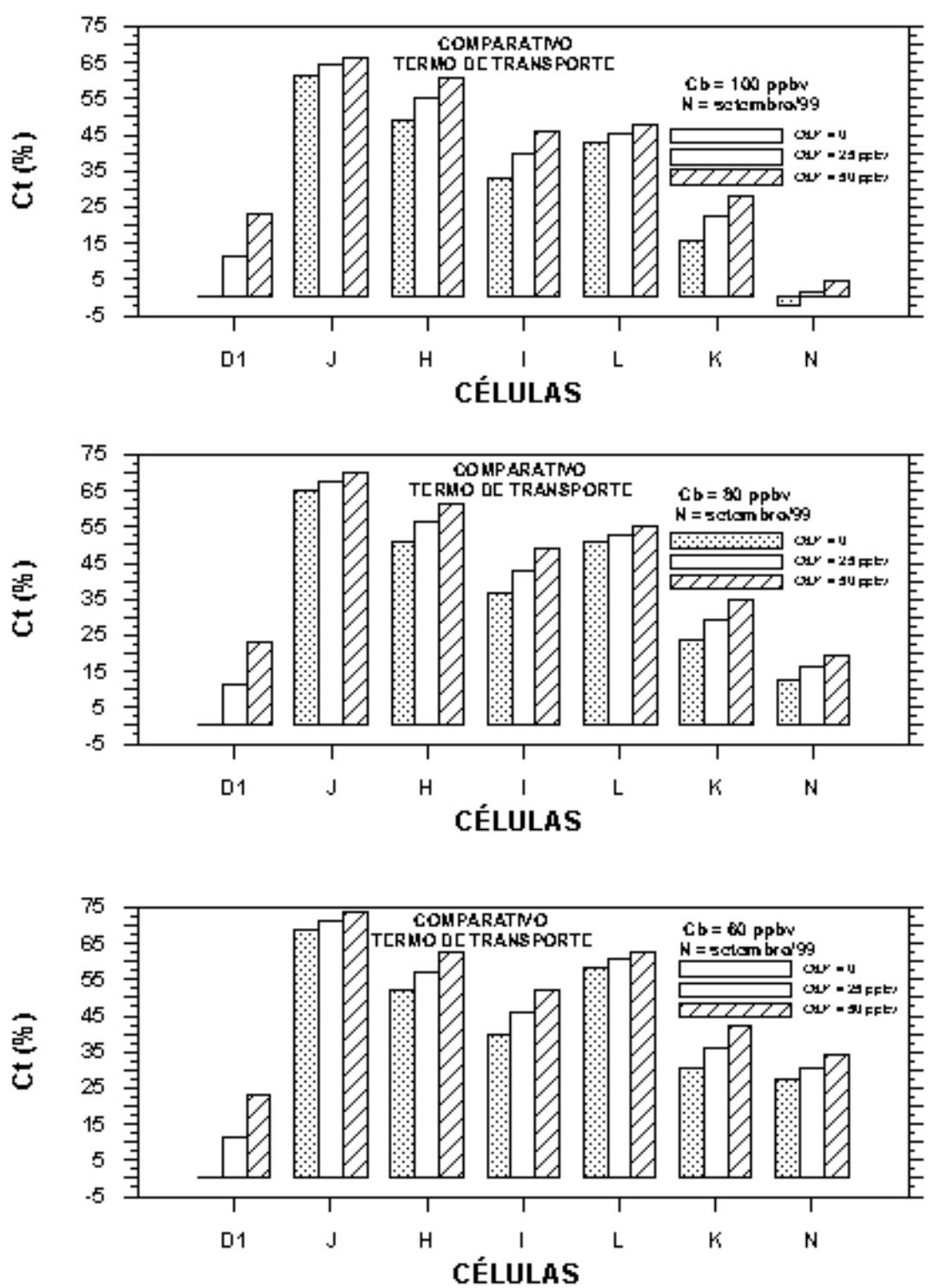

Figura 10 - Comparação entre os termos de transporte para diferentes condições de entrada para o mês de setembro, e Cb $=100,80$ e 60 ppbv respectivamente.

Figure 10 - Calculated transport term for three different input conditions for September, and Cb=100, 80 and 60 ppbv, respectively.

no eixo das abcissas mostramos os 3 casos de condições iniciais da célula D1.

Verificamos na Fig. 10 que os valores calculados não variam muito, mesmo com a variação grande imposta na condição básica que é o valor de $\mathrm{Cb}$ (entre 60 e 100). Além disto, os resultados (as 3 barras) também não variam muito com a variação de $\mathrm{CtD} 1$, que variou de um fator muito grande (de 0 a 50).

Com base nessa validação numérica do modelo, concluímos que o formalismo desenvolvido possibilita a determinação do termo de transporte, uma vez que os resultados são convergentes, isto é, mesmo variando bastante as condições de entrada do sistema de cálculo, os resultados não variam muito.
A partir destas condições de contorno definidas para a célula $\mathrm{D} 1$ e para $\mathrm{N}$ (número de focos de queimada) correspondente ao mês de setembro, obtém-se um $\mathrm{F}=0,9 \pm 0,2 \mathrm{ppbv}$ de $\mathrm{CO}$ por 100 focos (fator de conversão de focos de queimada para concentrações de $\mathrm{CO}$ em ppbv).

\section{RESUMO}

1- Foram feitos cálculos de trajetória para determinar o caminho de transporte continental de massas de ar na troposfera, em altitudes definidas, que mostraram a sua predominância na região leste e central do país; 
2- Foram analisados e ordenados dados de focos de queimada obtidos via satélites da série NOAA, para obter a distribuição espacial e temporal dos focos de queimada no Brasil;

3- Foram realizadas medidas aéreas de $\mathrm{CO}$ ao longo da área de estudo, a fim de verificar o transporte de contaminantes de áreas fonte para regiões não fonte de queimada;

4- Foi desenvolvida um formalismo para avaliar o termo de transporte de contaminantes de áreas fonte de queimada para não fonte, ao longo do corredor de circulação de massas de ar.

\section{CONCLUSÕES}

1- Medidas de CO a bordo de aeronave instrumentada mostraram em duas campanhas especiais grandes gradientes de concentração, como previsto. Os valores observados mostraram concentrações variando entre 100 a 600 ppbv.

2- Das condições de contorno obtém-se um fator regional de produção de $\mathrm{CO}$ de $0,9 \pm 0,2$ ppbv por 100 focos de queimada.

3- Calculou-se (com base nas condições de contorno da célula $\mathrm{D} 1$ e para $\mathrm{N}=$ número de focos de queimada de setembro) a proporção de CO que é importada de regiões fonte. Para a região J, que inclui o sul do Estado de Goiás e o norte do Estado de Mato Grosso do Sul, isto significa, para uma região com muito menos queimadas que o norte de Mato Grosso, por exemplo, uma injeção de até $70 \%$ do valor local observado de CO. Na região do Paraná, por exemplo, quase $50 \%$ do valor observado localmente é trazido para a região via transporte.

\section{AGRADECIMENTOS}

Agradecemos à FAPESP pelo financiamento parcial deste projeto. Agradecemos a infra-estrutura de todo o INPE, em especial aos pilotos do avião e ao pessoal do Laboratório de Ozônio. Ao CPTEC do INPE, em especial ao Dr. Igor Trosnikov.

\section{REFERÊNCIAS}

Austin, J. \& Tuck, A.F., 1985. The calculation of stratospheric air parcel trajectories using satellite data. Quart.J.R.Met.Soc., 111: 219-307.
Conte, S. D. \& Boor C. de, 1981. Elementary numerical analysis - An algorithmic approach. McGraw-Hill , 432p.

CPTEC. www.cptec.inpe.br ,1999. Mapa de focos de queimada.

Crutzen, P.J., Delany, A.C., Greenberg, J.,Haagenson, P., Heidt, L., Lueb, R., Pollock, W., Seiler, W., Wartburg, A. \& Zimmermann, P., 1985. Tropospheric chemical composition measurements in Brazil during the dry season. J.Atmos.Chem., 2: 233-256.

Delany, A.C., Haagenson, P., Walters, S., Wartburg, A.F. \& Crutzen, P.J., 1985. Photochemically produced ozone in the emission from large scale tropical vegetation fires. J.Geophys.Res., 90: 2425-2429.

Doty, B., 1995. The grid analysis and display system. GRADS Manual, 10 September, p. 148.

Doty, K. G. \& Perkey, D. J., 1993. Sensitivity of trajectory calculations to the temporal frequency of wind data. Monthly Weather Rev., 121: 387 401, February.

Fuelberg, H. E., Loring R. O. Jr., Watson, M. V., Sinha, M. C., Pickering, K. E., Thompson, A. M., Sachse, G. W., Blake, D. R. \& Schoeberl, M. R., 1996. TRACE A trajectory intercomparison 2. Isentropic and kinematic methods. J. of Geophys. Res., 101: n. D19, 23927-23939.

Kahl, J. D., Harris, J. M. \& Hebert, G. A., 1989. Intercomparison of three long-range trajectory models applied to Arctic haze. Tellus, 41 B: 524536.

Kirchhoff, V.W.J.H., 1996. Increasing concentrations of $\mathrm{CO}$ and $\mathrm{O} 3$, Env.Sci.Poll.Res., 3: 210-212.

Kirchhoff, V. W. J. H. \& Marinho, E. V. A., 1988. A concentração de monóxido de carbono (CO) no ar como parâmetro de pureza. Rev. Brasil. Geofís., 6(1): 9-16.

Kirchhoff, V.W.J.H. \& Marinho, E.V.A., 1989. A survey of continental concentrations of atmospheric CO in the Southern Hemisphere. Atmosph.Env., 23: 461-466.

Kirchhoff, V. W. J. H. \& Marinho, E. V. A., 1990. Surface carbon monoxide measurements in 
Amazonia. J. Geophys. Res., 95(D10): 1693316943.

Kirchhoff, V.W.J.H., \& Rasmussen, R.A., 1990. Time variations of $\mathrm{CO}$ and $\mathrm{O} 3$ concentrations in a region subject to biomass burning. J. Geophys. Res., 95: 7521-7532.

Kirchhoff, V. W. J. H. \& Alvalá, P. C., 1996. Overview of an aircraft expedition into the Brazilian cerrado for the observation of atmospheric trace gases. J. Geophys. Res., 101(D19): 2397323982.

Kirchhoff, V.W.J.H., Browell, E.V. and Gregory, G.L., 1988. Ozone measurements in the troposphere of an Amazonian rain forest environment. J. Geophys. Res., 93: 1585015860 .

Kirchhoff, V.W.J.H., Setzer, A.W. \& Pereira, M.C., 1989. Biomass burning in Amazonia. Geophys. Res. Lett., 5: 469-472.

Kirchhoff, V.W.J.H., Silva, I.M.O. \& Browell, E.V., 1990. Ozone measurements in Amazonia: dry season vs. wet season. J. Geophys. Res., 95: 16913-16926.

Longo, K. M., Thompson, A. M., Kirchhoff, W. J. H., Remer, L. A., Freitas, S. R. de, Dias, M. A. F. S., Artaxo, P., Hart, W. Spinhirne, J. D. \& Yamasoe, M. A., 1999. Correlation between smoke and tropospheric ozone concentration in Cuiabá during Smoke, Clouds and Radiation-Brazil (SCAR-B). J. Geophys. Res., 104(D10): 12113 - 12129.

Mariano, M. M., 1992. Estudo das Concentrações de Ozônio no Cerrado Brasileiro. São José dos Campos. 149 p. (INPE-5447-TDI/493). Dissertação (Mestrado em Geofísica Espacial) Instituto Nacional de Pesquisas Espaciais.

Marinho, E.V.A., Kirchhoff, V.W.J.H. \& Alvalá, P.C., 1997. Tropospheric nitrous oxide observations in Brazil: SCAR B mission, in SCAR B proceedings, ed. V.W.J.H.Kirchhoff, Transtec Editorial, ISBN 85-85417-09-9, pp129-132.

Merril, J.T., Black, R. \& Boudra, D., 1986. Techniques of Lagrangian trajectory analysis in isentropic coordinates. Month. Weath. Rev., 114: 571-581.

NCEP, 1998. http://wesley.wwb.noaa//ncep_data/ index.html. Dados meteorológicos.
Nobre, C. A., Mattos, L. F., Dereczynski, C. P., Tarasova, T. A. \& Trosnikov, I., 1998. Overview of atmospheric conditions during the Smoke, Clouds and Radiation-Brazil (SCAR-B) field experiment, J. Geophys., Res. 103(D24): 31809 - 31820, December 27.

Novelli, P. C.; Masarie, K. A. \& Lang, P. M., 1998. Distributions and recent changes of carbon monoxide in the lower troposphere, J. Geophys. Res., 103(D15): 19015 - 19033.

Pickering, K. E., Thompson, A. M., McNamara, D. P. \& Schoeberl, M.R., 1994. An intercomparasion of isentropic trajectories over the South Atlantic. Monthly Weather Rev., 122: 864-879.

Pickering, K. E., Thompson, A. M., McNamara, D. P., Schoeberl, M.R, Fuelberg, H. E., Loring Jr. R. O., Watson, M. V., Fakhruzzaman, K. \& Bachmeier, A. S., 1996. TRACE A trajectory intercomparison, 1, Effects of different input analyses. J. Geophys. Res., 101 (D19): 23909-23926.

Reichle, H.G.Jr., Connors, V., Holland, J.A., Hypes, W.D., Wallio, H.A., Casas, J.C., Gormsen, B.B., Saylor, M.A. \& Hesketh, W.D., 1986. Middle and upper tropospheric carbon monoxide mixing ratios as measured by a satellite borne remote sensor during November 1981. J.Geophys.Res., 91: 10,865-10,887.

Sachse, G. W., Harriss, R.C., Fishman, J., Hill, G. F. \& Cahoon D. R., 1988. Carbon monoxide over the Amazon Basin during the 1985 dry season. J.Geophys. Res., 93(D2): 1422-1430.

Satyamurty, P.; Nobre, C. A. \& Dias, P. L. S., 1998. Meteorology of the south hemisphere. Boston, Massachusetts: Am. Meteoro. Soc., December: 27(49): 119-139.

Seiler, W., 1974. The cicle of atmospheric CO. Tellus, n.2, pp. 116-135.

Seiler, W. \& Junge, C., 1970. Carbon monoxide in the atmosphere. J. Geophys. Res., 75(12): 22172226.

Setzer, A. W.; Pereira, M. C. \& Pereira A. C., 1992. O uso de satélites NOAA na detecção de queimadas no Brasil. Climanálise: 7(8): 41-53. 
Vianello, R. L. \& Alves, A. R., 1991. Meteorologia

básica e aplicações. Viçosa: Imprensa Universitária (UFV), 449p.
Williamson, D. L. \& Rasch, P.L., 1989. Twodimensional semi-Lagrangian transport with shape-preserving interpolation. Monthly Weather Rev: 117: 102-129.

\title{
NOTE ABOUT THE AUTHORS
}

\section{Cláudia Boian Aires}

Bacharel em Física pela Universidade Estadual Paulista (UNESP/Rio Claro), em fevereiro de 1991, Mestre em Geociências pela mesma instituição (julho de 1995) e doutora em Geofísica Espacial pelo Instituto Nacional de Pesquisas Espaciais (INPE), em março de 2001. Em maio de 2001 iniciou sua pesquisa de Pós-Doutorado intitulada: "Medidas de gases traço em regiões sob a influência do transporte de contaminantes de queimadas", junto ao Laboratório de Ozônio do INPE. Seu interesse de pesquisa envolve estudos relacionados à poluição atmosférica e ao transporte de poluentes, principalmente devido à queima de biomassa.

\section{Volker W.J.H.Kirchhoff}

Pesquisador do Instituto Nacional de Pesquisas Espaciais, INPE, desde 1969, é fundador e chefe do
Laboratório de Ozônio do INPE. Autor de mais de 80 publicações em revistas de circulação internacional, e de vários livros com tema principal sendo ozônio e queimadas na região Amazônica. Foi o coordenador brasileiro de vários experimentos científicos feitos em colaboração com a NASA, com grande participação de cientistas brasileiros e americanos, como o CITE 3, o TRACE-A e o SCAR-B. Recebeu o prêmio Group Achievement Award da NASA, por sua atuação destacada no projeto MAPS e o prêmio especial do Ministério do Meio Ambiente "em reconhecimento pelos relevantes trabalhos realizados para a proteção da camada de ozônio". Foi Coordenador de Ciências Espaciais, vice-Diretor, e Diretor interino do INPE.

\section{PÓS GRADUAÇÃO EM GEOFÍSICA ESPACIAL}

\author{
MESTRADO E DOUTORADO
}

Áreas de Pesquisa:

Alta Atmosfera, Eletricidade Atmosférica, Geomagnetismo, Ionosfera, Luminescência Atmosférica, Magnetosfera - Heliosfera, Média e Baixa Atmosfera

INPE - Serviço de Pós Graduação

Caixa Postal 515

12201-970 - São José dos Campos/SP

Fone: (12) 3945-6000

http://www.inpe.br 